\title{
Landeskundliche Wortschatzübungen auf der Basis von Kollokationen. Zur Nutzung von elexiko für DaF/DaZ-Lehrende
}

\begin{abstract}
Dieser Beitrag geht der Frage nach, wie elexiko als eine Grundlage für Wortschatzübungen im Deutsch als Fremdsprache (bzw. Zweitsprache) Unterricht genutzt werden kann. Ausgegangen wird dabei davon, dass die explizite Wortschatzarbeit im Rahmen von Sprachunterricht, besonders gepaart mit einer gelungen vermittelten sprachbezogenen Landeskunde, das Verstehen der Sprache und die Fähigkeit zur erfolgreichen Kommunikation fördert. Dies setzt voraus, dass Deutschlehrende mit relevantem Sprachmaterial arbeiten, das sich möglichst eng am authentischen Sprachgebrauch orientiert und kulturelles Wissen mit transportiert. Hier bieten korpusgestützt erarbeitete Wörterbücher eine nützliche Quelle. Am Beispiel der im Wörterbuch aufgeführten Kollokationen wird skizziert, wie die Angaben aus diesem Bereich von Deutschlehrenden gewinnbringend für die Erarbeitung von Wortschatzübungen genutzt werden könnten.

This article addresses the question of how elexiko can be used as a basis for vocabulary exercises in German as a Foreign (or Second) Language instruction. The starting point is that explicit vocabulary work in the context of language teaching, especially when paired with successful language-related teaching of culture and society, promotes comprehension of the language and the ability to communicate successfully. This requires German teachers to work with relevant language material, which is based as closely as possible on authentic language use and which transmits cultural knowledge. Corpus-based dictionaries are a useful source for this. Using the example of collocations listed in the dictionary, the article outlines how this type of information can be profitably utilised by German language teachers for the development of vocabulary exercises.
\end{abstract}

\section{Einleitung}

Wie kann elexiko (bzw. können vergleichbare Wörterbücher) für die Konzipierung von landeskundlichen Wortschatzübungen im Sprachunterricht ${ }^{1}$ genutzt werden? Diese Frage steht in diesem Beitrag im Mittelpunkt der Betrachtung. Eine allumfassende Beantwortung ist hier allerdings nicht möglich. Kleine Beobachtungen anhand einiger ausgewählter Wortartikel aus elexiko (und anderen Wörterbuchquellen) sollen ein Annäherungsversuch zur Beantwortung der Frage in diesem Beitrag sein (vgl. Kapitel 3.2). Ausgewählt wurden hierfür einige Wortartikel, die landes- und kulturkundliche Sichtweisen und Besonderheiten rund um das Thema ,Zeit' vermitteln. Die Kenntnis hierüber ist für Lerner einer Sprache insbesondere als Zweit- bzw. Fremdsprache von großer Bedeutung (vgl. Maijala 2009; Simon-Pelanda 2001, S. 932f.), weil es sich dabei um für den Alltag hochrelevantes Wissen handelt. Wissen über Wortschatz sollte daher von Lehrenden in Wortschatzübungen (in expliziter oder impliziter Form) in den Unterricht integriert werden (vgl. auch Petelin 2011). Insbesondere die Kollokationsangaben zu einem Stichwort aus einem Wörterbuch wie elexiko - so wird in diesem Beitrag angenommen - können dabei als Anregung verwendet werden. Unter Kollokationen werden die unmittelbaren, statistisch signifikanten Kontextwörter verstanden, die mit dem Stichwort gemeinsam auftreten. Diese deuten häu-

\footnotetext{
Angenommen werden soll in diesem Beitrag ein Lerner des Deutschen als Fremd- bzw. Zweitsprache, der sich nach dem Europäischen Referenzrahmen auf dem Niveau zwischen B1 und C1 (also zwischen fortgeschrittener und fachkundiger Sprachverwendung bzw. -kenntnis) bewegt. Vergleiche hierzu im Detail die Ausführungen zu den einzelnen Niveaustufen im Europäischen Referenzrahmen.
} 
fig auf bestimmte Kontexte und Diskurse hin, die auch landes- und kulturspezifische Details enthalten. Daher scheinen sie bei der Vermittlung solcher Aspekte von besonderer Relevanz.

Was kann in diesem Zusammenhang unter ,Landeskunde“ verstanden werden? Unter Landeskunde wird im Allgemeinen Folgendes zusammengefasst: „Wirtschaft, Gesellschaft, Kultur, Sport, Geografie, aktuelle Politik, Geschichte, die Art und Weise, wie Personen in deutschsprachigen Ländern miteinander kommunizieren, die sogenannten typischen Eigenschaften von Vertretern des deutschsprachigen Raums [...]" (Rösler 2012, S. 195). Das Wissen über Landeskunde spielt generell für jedes Mitglied der Gesellschaft, aber ganz besonders im Zusammenhang mit dem Erwerb einer fremden Sprache eine wichtige Rolle.

Der lebensweltliche Erwerb von Landeskunde bezogen auf den Kontext, in dem man lebt, ist eine stetig wachsende Mischung aus in Bildungsinstitutionen und durch individuelles Erleben von und Interaktion mit Welt erworbenem Wissen. (ebd., S. 196)

Befragt man Erwachsene im universitären Kontext zu ihren Erwartungen in Bezug auf die Behandlung der landeskundlichen Inhalte im DaF/DaZ-Unterricht, wie das beispielsweise Maijala (2009) getan hat, ${ }^{2}$ wollen diese insbesondere etwas über das alltägliche Leben erfahren. ${ }^{3}$ Dies unterstützt auch Rösler:

Der Fremdsprachenunterricht muss sich bis zu einem gewissen Grad auf ein alltagsprachlich geläufiges Konzept von Kultur einlassen, das davon ausgeht, dass es so etwas wie eine homogene, in sich geschlossene und auf nationale Gruppen bezogene Kultur gibt. Dem gegenüber steht, dass jede dieser sogenannten nationalen Kulturen aus einer Ansammlung von Menschen besteht, die aus sehr unterschiedlichen Kontexten zusammengekommen sind, wie z. B. die Migrationsgeschichte in Deutschland der letzten 50 Jahre zeigt [...]. (Rösler 2012, S. 209)

Die Lehrenden stehen wegen der Erwartungen und der meist sehr unterschiedlichen Voraussetzungen der Lernenden vor einer Herausforderung, da sie sich für die Vermittlung von landeskundlichen Themen und Inhalten grundsätzlich darauf einstellen müssen,

dass die Vorstellung von einer homogenen Kultur zwar ein offensichtlich unvermeidliches Hilfsmittel ist, wenn man sich in räumlich beschränkten Lehrwerken und zeitlich beschränkten Unterrichtssituationen an das Anderssein des zielkulturellen Raums heranwagt. (Rösler 2012, S. 209)

Die Lehrperson steht also vor der Aufgabe, in der beschränkten Unterrichtssituation einerseits ein angenommenes homogenes Bild einer Kultur zu vermitteln und gleichzeitig aber mit zu berücksichtigen, ,,dass jeder Mensch ein individuelles Bild einer bestimmten Kultur beschreiben kann“" (Rösler 2012, S. 209).

Hinter Begriffen wie ,kulturspezifisch` bzw. ,Kultur` verbergen sich dabei vielschichtige Konzepte und Theorien. In einer ersten Annäherung kann unter ,Kultur` nach Hansen (2000) Folgendes verstanden werden:

2 Im Rahmen des von Maijala (2009) untersuchten universitären Deutschkurses wurde das DaF-Lehrwerk „Passwort Deutsch 3. Kurs- und Übungsbuch“ (2002) vom Klett Verlag verwendet. In der Befragung wurde die Vermittlung von Landeskunde im Deutschunterricht untersucht. „Für diesen Zweck wurde im Deutschkurs auf dem Niveau A2 des Europäischen Referenzrahmens eine Umfrage durchgeführt, an der insgesamt 48 Deutschlernende teilnahmen“" (Maijala 2009, S. 377).

3 Auf die Frage „Was möchten die Lernenden von der fremden Kultur wissen?“ war die Antwort „Alltägliches Leben“" am häufigsten hinter „Traditionen“, „Gesellschaft und Politik“, „Sehenswürdigkeiten/ Geographie“, „Geschichte“ und anderen Bereiche (vgl. Tabelle 1 in Maijala 2009, S. 378). 
Kultur meint die Veränderung der Natur durch menschliche Tätigkeit, was dazu führt, daß die natürliche Ordnung durch eine vom Menschen geschaffene ersetzt wird. Gleichzeitig berücksichtigt diese Bedeutung, daß diese Ersatzordnung bei verschiedenen Völkern verschieden ausfällt. Zunächst ohne jede Wertung sprechen wir deshalb von fremder und eigener Kultur. (Hansen 2000, S. 15)

Im Folgenden wird erörtert, wie ein Lehrender ein Wörterbuch wie elexiko dazu benutzen kann, Landeskunde und insbesondere eine sprachbezogene Landeskunde (vgl. Bettermann 2010) an Deutschlernende zu vermitteln. Dies geschieht nicht pauschal, sondern an ausgewählten Beispielen. Anhand des Angabebereichs „Kollokationen“ in elexiko (vgl. Kapitel 3.1.4) soll exemplarisch herausgearbeitet werden, wie aus den korpusgeleitet erarbeiteten Angaben sprachbezogene Wortschatzübungen für den landeskundlichen und auf das alltägliche Leben bezogenen Unterricht entwickelt werden könnten. Als potenzielle Personengruppe für einen solchen Nutzen werden besonders DaF/DaZ-Lehrende angenommen. Die Aspekte Kultur und Sprache sollen als eng miteinander verzahnte Bereiche verstanden werden. Die Grenzziehung zwischen Weltwissen und Sprachwissen verläuft mitunter fließend. ,Nicht die Kultur bereichert die Sprache, sondern die Sprache ist als eine Art Bereicherung der gesamten menschlichen Kultur zu betrachten" (Grucza 2000, S. 23).

\section{Wortschatzarbeit und sprachbezogene Landeskunde}

Es bestand in Hinblick auf den Bereich Wortschatzarbeit im Sprachunterricht lange Zeit die Sichtweise, dass Wortschatz bzw. Wörter (im Allgemeinen) nebenbei mitgelernt werden (vgl. Hochstadt/Krafft/Olsen 2013, S. 185). Ein defizitärer Wortschatz wurde in der Diskussion um Bildungsstandards gar nicht wirklich wahrgenommen. „Dementsprechend wird der Zugriff auf ein differenziertes mentales Lexikon - wie man das begriffliche Netz ,im Kopf' nennt - auch in der Schule vorausgesetzt" (ebd., S. 185).

Für den Unterricht bedeutet solch eine Sichtweise im Umkehrschluss, dass expliziter Wortschatzarbeit im Grunde gar kein Raum zugesprochen wird. Die sogenannte ,Kognitive Wende' in Psychologie und Linguistik hat die lexikalische Semantik stärker in den Vordergrund gestellt, was letztlich auch dazu geführt hat, dass die Bedeutung der Wortschatzarbeit für den Sprachunterricht wiederentdeckt wurde (vgl. hierzu auch Ulrich 2011, S. 9) und sich in verschiedenen Strömungen in der Wortschatzarbeit niedergeschlagen hat (vgl. Hochstadt/Krafft/Olsen 2013, S. 190ff.). ${ }^{5}$ So stellt Ulrich (2007, S. 34) fest: „Es erscheint eben doch sinnvoll, den Wortschatzerwerb der Schüler durch direkte Wortschatzübungen zu unterstützen." Wird über ,direkte", ,explizite' Wortschatzarbeit gesprochen,

4 Vor dem Hintergrund der Definition von ,Kultur' im Zitat von Hansen (2000) und der Datenbasis, auf der die Angaben in elexiko ermittelt werden, sind noch zwei Aspekte zu berücksichtigen: Erstens ist zu beachten, dass die Daten, die in den elexiko-Wortartikeln zusammengetragen sind, natürlich ein Szenario für das Wortfeld ,Zeit' im deutschsprachigen Raum entwerfen (das elexiko-Korpus enthält Texte aus deutschen, österreichischen und Schweizer Zeitungen), es gilt also prinzipiell nicht allerorts bzw. global. Zweitens kommen genau aufgrund der Zusammenstellung des elexiko-Korpus ausschließlich aus Pressetexten auch teils Thematisierungen in einzelnen Wortgruppen vor, die über das Deutschsprachige hinausgehen (vgl. z.B. in Tabelle 2 die Kollokationen Sachschaden und Schüsse zum Stichwort Nacht, bei denen man einen direkten Zusammenhang der Berichterstattung in Zeitungstexten vermuten kann).

5 Hochstadt/Krafft/Olsen (2013, S. 190ff.) beschreiben vier Strömungen in der Wortschatzarbeit: die traditionelle, die lexikonorientierte, die textorientierte Wortschatzarbeit und das robuste Wortschatztraining. Vgl. hierzu auch die Ausführungen von Töpel in diesem Band bzw. zur Wortschatzdidaktik in Reder (2009). 


\section{Christine Möhrs}

so steht hinter dieser Perspektive immer das Konzept des Sprachbewusstseins (vgl. language awareness in Ulrich 2007, S. 35, 2011, S. 15). Es kann und sollte davon ausgegangen werden, ,dass ein im Unterricht bewusst gemachtes, erhelltes und durchschautes semantisches Netzwerk besser verfügbar ist als ein undurchschautes" (Ulrich 2007, S. 35) und dass eine , explizite' Wortschatzarbeit somit zum besseren und erfolgreicheren Sprachlernen führen kann. Welcher Strömung ein Lehrender bei der Konzeption und Umsetzung von Wortschatzübungen prinzipiell folgt, hängt vermutlich von verschiedenen Faktoren ab (z.B. von dem Sprachniveau der Lernenden oder dem im Unterrichtskontext behandelten Thema). Man kann mit Ulrich (2011) aber in jedem Fall festhalten, dass

lexikalisches Lernen [...] als ein interaktiver Prozess zu verstehen ist, der erstens durch sprachliche Impulse von außen, zweitens durch vorhandene allgemein-kognitive Fähigkeiten des Lernenden und drittens durch erworbene lexikalische Erwerbsstrategien gesteuert wird. (ebd., S. 19)

Wird über ein bestimmtes Themengebiet implizit auch ein Teil kultur- und landeskundliches Wissen vermittelt, so kann man am ehesten von ,sprachbezogener Landeskunde" nach Bettermann (2010) sprechen. Er versteht unter ,sprachbezogener Landeskunde' einen Oberbegriff,

unter dem integrative Unterrichtskonzepte für Deutsch als Fremd- und Zweitsprache zusammengefasst werden, welche die Berücksichtigung des Zusammenhangs von Sprachenlernen und Kulturvermittlung/ Kulturverstehen im Fremdsprachenunterricht [...] zum zentralen Anliegen haben. Eine solche Landeskunde ist kommunikativ und interkulturell konzipiert und unterscheidet sich in dieser Akzentuierung von einer eher explizit angelegten, gegenstandsbezogenen oder auch problemorientierten Landeskunde. (ebd., S. 1454)

Diese nach Bettermann beschriebene ,sprachbezogene Landeskunde' basiert auf der in Kapitel 1 erläuterten Idee von Landeskunde, fokussiert aber weniger auf landeskundliches Faktenwissen ${ }^{6}$, sondern besonders auf sprachliches Wissen. Das Wissen um landeskundliche Aspekte und auch landesspezifisches sprachliches Wissen können bei einer erfolgreichen Aneignung zum kulturellen Verstehen der erlernten Sprache und Kultur beitragen (vgl. Bettermann 2010, S. 1454; Simon-Palanda 2001). Durch die Stärkung der Wortschatzarbeit im Allgemeinen (s.o.) und die bewusste Vermittlung landeskundlicher Aspekte (vgl. Koreik 2011) vor allem in kommunikationsorientiert-interkulturellen Ansätzen rückt die Erkenntnis in den Blick, dass „Verständigungshandlungen nur gelingen können, wenn neben sprachlichen Wissensbeständen gleichzeitig ein spezifisches Kontext- und Interaktionswissen vermittelt wird" (Veeck/Linsmayer 2001, S. 1165). Bettermann fasst daher zusammen, dass es bei sprachbezogener Landeskunde in methodischer Hinsicht darum geht, den „Erwerb von Strategien und Techniken zur Erschließung der in Sprache gefassten landeskundlichen Elementen als Beitrag zur Entwicklung der interkulturellen Kompetenz durch die Lernenden" (Bettermann 2010, S. 1455) in den Mittelpunkt zu stellen.

\section{Von der Kollokation zur Wortschatzübung}

Bei Wortschatzübungen insbesondere für Lerner des Deutschen als Fremd- bzw. Zweitsprache ist es von großem Interesse, dass den Lernenden ihrem Sprachniveau angepasste Wortschatzübungen an die Hand gegeben werden. Gleichzeitig ist es für Lerner von höchstem Interesse, nicht einen für sie irrelevanten Wortschatz zu lernen, sondern viel-

Zu Übungen (und Materialien) in Hinblick auf landeskundliches Faktenwissen vgl. z.B. die vom Goethe-Institut entwickelten und auf den Webseiten bereitgestellten Angebote: www.goethe.de/de/spr/unt/ kum.html. 
mehr einen Wortschatz, der auf authentischem Sprachmaterial basiert, damit usuelle Muster und gängige Kontexte illustriert werden, und der somit relevant für sie ist. Umfangreiche Textkorpora (wie z.B. das Deutsche Referenzkorpus DEREKo des Instituts für Deutsche Sprache) bieten eine große Menge authentischen Sprachmaterials an, die prinzipiell als Fundus an Sprachdaten auch für Wortschatzübungen denkbar wären. Bevor man allerdings auf die Daten aus einem umfangreichen Korpus zugreifen kann, gilt es, die Abfragesprache der jeweiligen technischen Umgebung sowie die Funktion der Korpusanalysetools genau zu kennen, anwenden und letztlich deuten zu können, um sie in einem nächsten Schritt in eine Wortschatzübung, übersetzen` zu können. Es mag für eine Lehrperson, die in einen engen, unterrichtlichen Kontext eingebunden ist, eine Hürde sein, Wortschatzübungen auf der Grundlage von großen allgemeinsprachigen Textkorpora ohne spezielle linguistische Annotationen selbst zu erarbeiten. Korpusgestützt erarbeitete, allgemeinsprachige Wörterbücher könnten daher eine nützliche Grundlage für Lehrende sein, um Kollokationen zu Wörtern aufzufinden, um damit Wortschatzübungen für Lehrkontexte $\mathrm{zu}$ entwickeln. Generell sind als Unterrichtsinstrumente auch Wörterbücher an sich zu nennen, besonders in Verbindung mit den neuen Medien (vgl. hierzu auch Müller/Siever 2011). Besonders für Onlinewörterbücher gilt: „Aspekte wie Echtzeit-Zugriff, Unabhängigkeit von Ort und Zeit ermöglichen es, dass kollaborativ an einem Wörterbuch gearbeitet werden kann - ein Aspekt, der auch bei einem Unterrichtsprojekt von Vorteil ist" (ebd., S. 44). Ob die Erarbeitung von Wortschatzübungen auf der Basis von Wörterbüchern prinzipiell möglich ist, soll im Folgenden anhand von Beispielen erörtert werden.

Als Beispielwortgruppen mit den entsprechend dazugehörigen Wortartikeln wurden exemplarisch Bezeichnungen für Tageszeiten (morgens, Morgen, mittags, Mittag, nachmittags, Nachmittag, abends, Abend, nachts, Nacht) in verschiedenen Wörterbüchern (siehe 3.1.1 bis 3.1.4) betrachtet. Die Wortartikel können unter dem Thema ,Zeit' gruppiert und damit auch als Grundlage für implizite landeskundliche Wortschatzförderung (vgl. Kap. 2) herangezogen werden. Denn die sprachlichen Kontexte und ,die Gebrauchsbedingungen für Wörter und Lexeme, die in den Wörterbüchern dokumentiert oder auch in elektronischen Korpora nachvollziehbar gemacht worden sind, spiegeln Vorschläge für die Sicht auf bestimmte Welt-Konstellationen“ (Eichinger 2008, S. 4f.).

\subsection{Kollokationsangaben in (Online-)Wörterbuchressourcen}

Eine kleine Sichtung verschiedener (Online-)Wörterbuchressourcen zeigt, dass Kollokationen dem Nutzer in Wörterbüchern ganz verschieden und in unterschiedlichem Umfang dargeboten werden.

\subsubsection{Duden online}

Das Wörterbuch „Duden online“ präsentiert sogenannte ,Wortwolken“ unter der Rubrik „Typische Verbindungen“7 (in Abb. 1 ist eine Wortwolke mit adjektivischen Kollokatoren zum Stichwort Morgen zu sehen). Diese computergenerierten Wortwolken bieten dem

\footnotetext{
In den Online-Benutzerhinweisen von „Duden online“ (www.duden.de) ist hierzu zu lesen: „Die typischen Verbindungen sind computergeneriert und zeigen in Form von Wortwolken den Kontext, in dem das Stichwort in Texten (Sätzen) typischerweise vorkommt. Die Textgrundlage bildet das Dudenkorpus. [...] Die Daten sind maschinell erzeugt, die Berechnung basiert auf statistischen Verfahren. Die Wortwolke veranschaulicht den Grad der Typizität zwischen dem (schwarzen) Stichwort und den (roten) Kontextwörtern. Größer dargestellte Kontextwörter gehen dabei eine typischere Verbindung mit dem
} 
Nachschlagenden die adjektivischen, verbalen oder substantivischen Kollokatoren zu einem Stichwort an. Bei polysemen Stichwörtern gewährleistet dieser automatisierte Prozess aber keine kontextuelle Disambiguierung der Partnerwörter. Auffällig beim Stichwort Morgen (Abb. 1) sind beispielsweise die Kollokatoren Mannheimer und rheinisch bzw. graduiert, die sich nicht auf Morgen in der Lesart, Tageszeit' beziehen, sondern z.B. Teil von Namen von Tageszeitungen (Mannheimer Morgen) sind. An diesem Beispiel zeigt sich, dass es (insbesondere bei polysemen Wörtern) sehr sinnvoll erscheint, die Kollokatoren lesartenbezogen anzugeben.

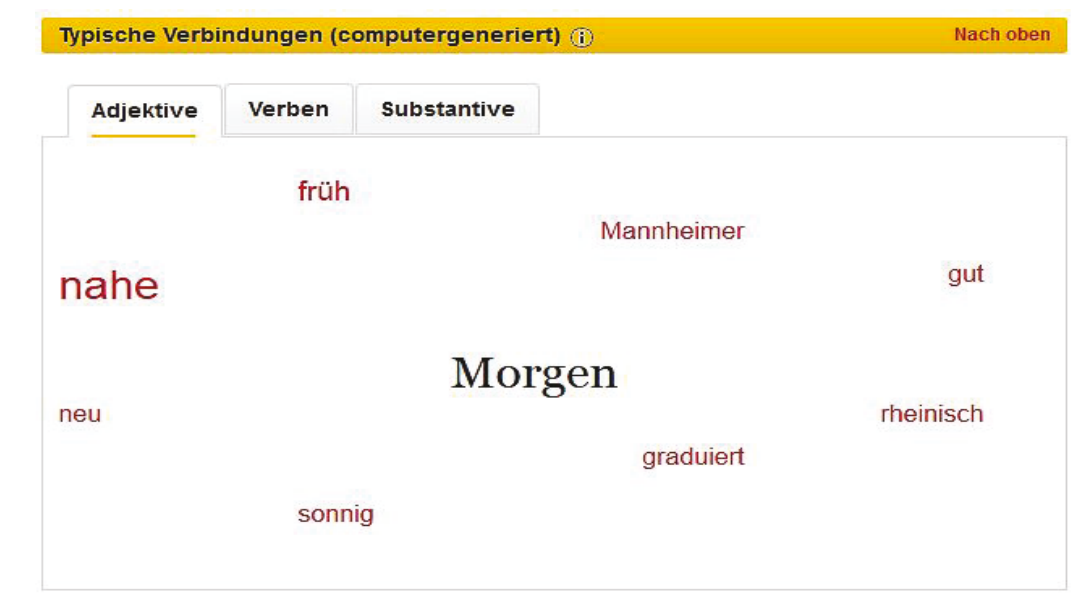

Abb. 1: Wortwolke der Adjektivkollokatoren zum Stichwort Morgen (Duden online)

\subsubsection{Digitales Wörterbuch der deutschen Sprache (DWDS)}

Ebenfalls in Form einer Wortwolke werden dem Nutzer im DWDS Kollokationen präsentiert, im ersten Schritt wortartenübergreifend (vgl. Abb. 2). Übertitelt ist dieser Bereich mit „DWDS-Wortprofil““ (vgl. Geyken 2011a und 2011b). Dieses ,ist das Ergebnis einer automatischen syntaktischen und statistischen Analyse sehr großer Korpora. Es liefert einen kompakten Überblick über die statistisch signifikanten syntagmatischen Beziehungen eines Wortes mit anderen Wörtern“(Geyken 2011b, S. 118).

Stichwort ein als Wörter in kleinerer Schriftgröße. Die Wortwolken sind nach Wortarten gruppiert und über die Reiter „Adjektive“, „Verben“ und „Substantive“ zugänglich. Da die maschinelle Analyse nicht zwischen gleich geschriebenen Wörtern mit unterschiedlichen Bedeutungen (z.B. [der] Kiefer vs. [die] Kiefer) unterscheiden kann, zeigt die Wortwolke mitunter Wörter aus dem Kontext beider Wörter und nicht nur aus dem Kontext des ausgewählten Stichworts. In Einzelfällen können von der Normschreibung abweichende Verbindungen zur Anzeige kommen. Die bereitgestellten Daten erheben keinen Anspruch auf Vollständigkeit. Sie sind kein Ergebnis redaktioneller Arbeit und stellen keine Handlungsempfehlung oder Wertung dar.“ (www.duden.de/hilfe/typische-verbindungen) 


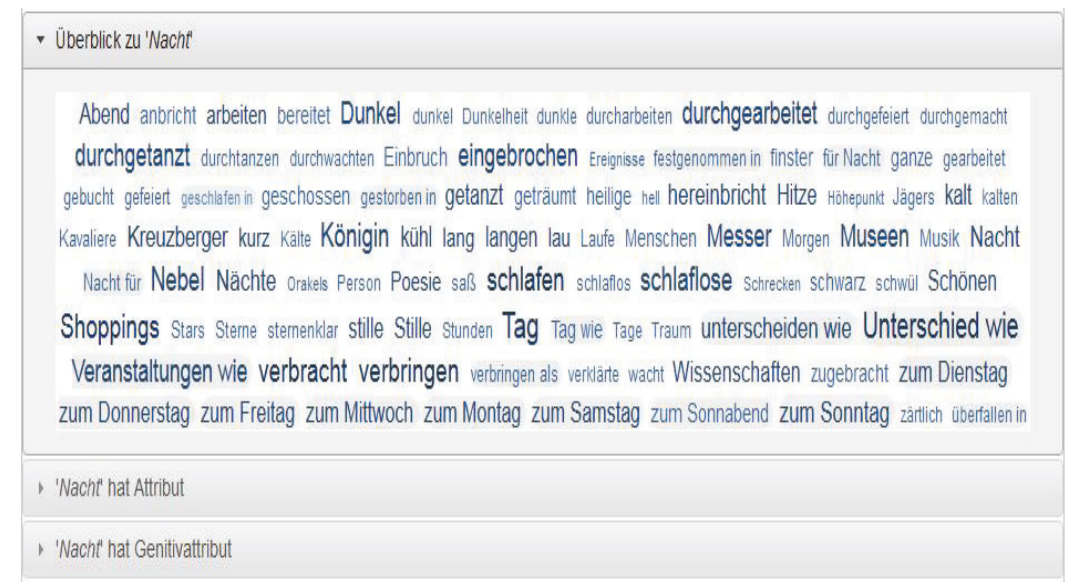

Abb. 2: Wortwolke mit Kollokatoren zum Stichwort Nacht (DWDS)

Diese Kollokationen sind wie bei „Duden online“ nicht nach Lesarten sortiert. Neben einem wortartunabhängigem Gesamtüberblick über alle Kollokatoren (wie in Abb. 2 gezeigt) stehen je nach Wortart des jeweiligen Stichwortes Wolken zur Verfügung, die nach syntaktischen Gesichtspunkten (z.B. ",Stichwort" hat Attribut", ,,Stichwort" ist Genitivattribut von“ etc.) sortiert sind (vgl. in Abb. 2 unter dem Kasten „Überblick“).

\subsubsection{Kollokationenwörterbuch}

Im „Kollokationenwörterbuch“ sind die Kollokatoren, ähnlich wie im DWDS, nach syntaktischen Kriterien zu den einzelnen Stichwörtern aufgeführt. Die Angaben in diesem Spezialwörterbuch sind im Gegensatz zu den Angaben in „Duden online“ oder DWDS redaktionell erarbeitet. ${ }^{8}$ Die auf der Basis verschiedener deutschsprachiger Korpora ${ }^{9}$ zusammengestellten Kollokatoren zu den Stichwörtern sind in einem „,manuellen Analyseprozess auf die artikelrelevanten Daten reduziert und systematisiert ${ }^{" 10}$ worden. Neben der Datenerhebung mit Hilfe korpusanalytischer Methoden erfolgt der Prozess der Erarbeitung der Angaben in einer, ,anschliessenden lexikographischen Aufbereitung der Daten unter pragmatisch-semantischen Gesichtspunkten“"11. Im Ergebnis werden dem Nutzer dann die Kollokatoren zu einem Stichwort nach Wortarten gruppiert präsentiert (vgl. Abb. 3), die zusätzlich durch Beispielsätze und Zusammensetzungen ergänzt sind.

8 Diese Online-Anwendung präsentiert einen Teilausschnitt aus dem Wörterbuch „Feste Wortverbindungen des Deutschen - Kollokationenwörterbuch für den Alltag“ (Häcki Buhofer et al. 2014). Besonders interessant im Kontext dieses Beitrags ist, dass zusätzlich zu dem Wörterbuch ein „Beiheft für Selbststudium und Unterricht“" (Meier et al. 2014) mit Wortschatzübungen erschienen ist, die als Orientierung für Lehrende bei der Konzipierung von Wortschatzübungen dienen sollen (vgl. Meier/Widmer 2014). Hier zeigt sich ebenfalls die Idee, dass auf der Basis von Kollokationen Wortschatzübungen erstellt werden können und dass Wörterbücher (hier im Speziellen ein Kollokationenwörterbuch) dazu eine Datengrundlage liefern können. Allerdings steht hier nicht die Vermittlung von sprachbezogener Landeskunde im Mittelpunkt.

9 Vgl. zu den verwendeten Korpora: http://colloc.germa.unibas.ch/web/projekt/korpus/.

10 http://colloc.germa.unibas.ch/web/projekt/korpus/.

11 http://colloc.germa.unibas.ch/web/projekt/kollokationen/. 
KDLLDKATI $\square$ NENUDERTERBUCH

Typische und gebräuchliche Wortverbindungen des Deutschen

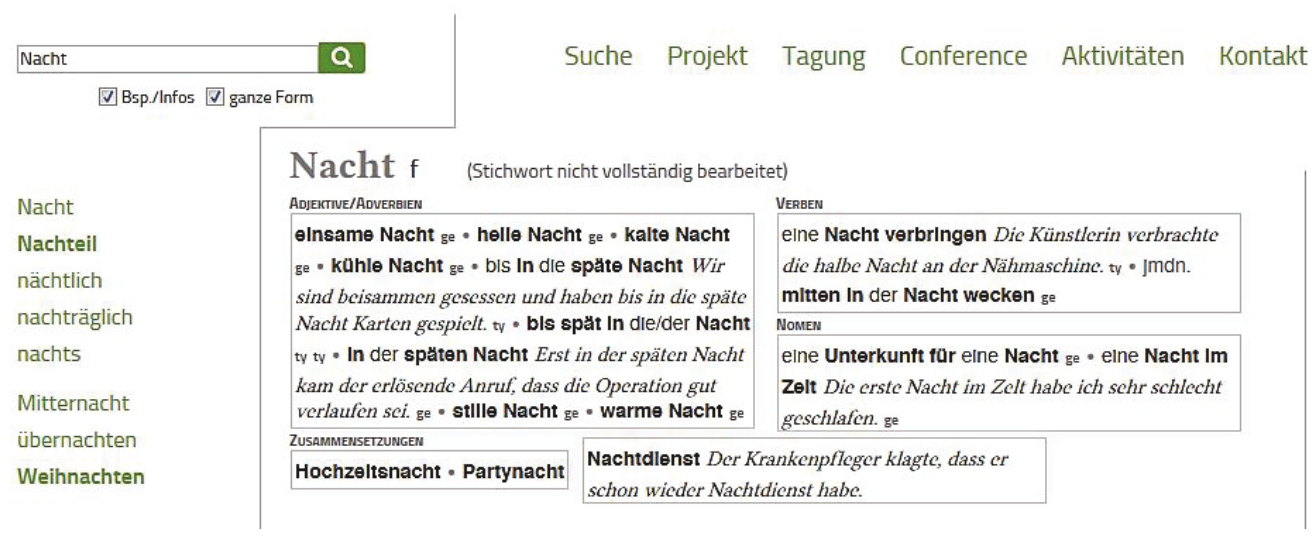

Abb. 3: Kollokationen zum Stichwort Nacht (Kollokationenwörterbuch)

\subsection{4 elexiko}

Bei dem Wörterbuch elexiko handelt es sich um ein Onlinewörterbuch, das in der Beschreibung und Verwendung der Bedeutung und des Gebrauchs der deutschen Gegenwartssprache seinen Schwerpunkt hat. Das Wörterbuch ist ausschließlich für das Internet konzipiert und die Angaben darin sind vollständig neu erarbeitet. Die Konzeption des Wörterbuches und seiner verschiedenen Angabebereiche ist im Sammelband Haß (Hg.) (2005) dargelegt und in zahlreichen Projektpublikationen ${ }^{12}$ beschrieben. In einem zweiten größeren Sammelband wurden Reflexionen über die lexikografische Arbeit im Projekt festgehalten, der - laut Titel - vor allem „Erfahrungsberichte aus der lexikografischen Praxis eines Internetwörterbuchs“ (Klosa (Hg.) (2011)) präsentiert. In Bezug auf grundsätzliche, konzeptionelle Überlegungen sei auf die beiden Sammelbände verwiesen, im Folgenden werden nur einige zentrale Aspekte zum Angabebereich der Kollokationen ausgeführt.

Alle Angaben in elexiko sind auf der Basis eines eigens für lexikografische Zwecke zusammengestellten elexiko-Korpus (vgl. Storjohann 2005) erarbeitet. Es handelt sich bei der Datenbasis also um umfangreiches authentisches Sprachmaterial, das eine empirische Auswertung des Sprachgebrauchs ermöglicht. Neben Angabebereichen, die aus der Tradition der Printlexikografie bekannt sind (wie „Sinnverwandte Wörter" und „Grammatik“), gibt es in elexiko den Angabebereich „Kollokationen“, der in der in elexiko dargebotenen Form als ein „lexikografisches Novum“ (Klosa/Storjohann 2011, S. 49) bezeichnet werden kann. In diesem Bereich wird die kontextuelle Umgebung des Stichwortes gezeigt und es wird illustriert, ,wie sich um das Stichwort als Zentrum [...] [ein] Netz zur semantischen Umgebung auf syntagmatischer Ebene“ (ebd., S. 49) anordnet. Die Kollokationen „sollen einen bestimmten Typ kognitiver Assoziationen zwischen dem Stichwort in einer Lesart und Wörtern in dessen Textumgebung darstellen" (Haß 2005a, S. 228). Sie werden lesartenbezogen in Form von „Fragen und zugehörigen Antworten (in Form von Wortlis-

12 Vgl. hierzu auch: www.owid.de/wb/elexiko/projekt/pgProjektveroeffentlichungen.html. 
ten) geordnet und erscheinen online als solche Gruppen linear untereinander" (Klosa/Storjohann 2011, S. 49) (vgl. das Beispiel in Abb. $4^{13}$ ).

Für die Angabe der Kollokationen werden die statistisch signifikanten Kookkurrenzpartner zu einem Stichwort ausgewertet. Den Lexikografinnen und Lexikografen steht dazu die Kookkurrenzanalyse (Belica 1995) des Korpusanalysetools COSMAS II zur Verfügung. Die Kollokatoren werden nach automatischer Analyse redaktionell ausgewertet, interpretiert und kontextuell zugeordnet. Neben der Gruppierung der Kollokatoren durch die Lexikografen des Projektes werden sie beispielsweise auch zur Illustrierung in der Bedeutungserläuterung aufgegriffen, sodass die verschiedenen Angabebereiche in elexiko stark miteinander verzahnt sind.

Die ursprüngliche Idee bei der Konzeption von elexiko war, dass sowohl Muttersprachler wie zumindest in eingeschränktem Maße nichtmuttersprachliche Deutschlerner, ,in einem späteren Lernstadium“ (Haß 2005a, S. 6) als Nutzergruppe für das Wörterbuch in Frage kommen sollen. Daneben soll es als Wort-Datenbank, also als Instrument für die Wortschatzforschung, dienen (vgl. ebd., S. 1).

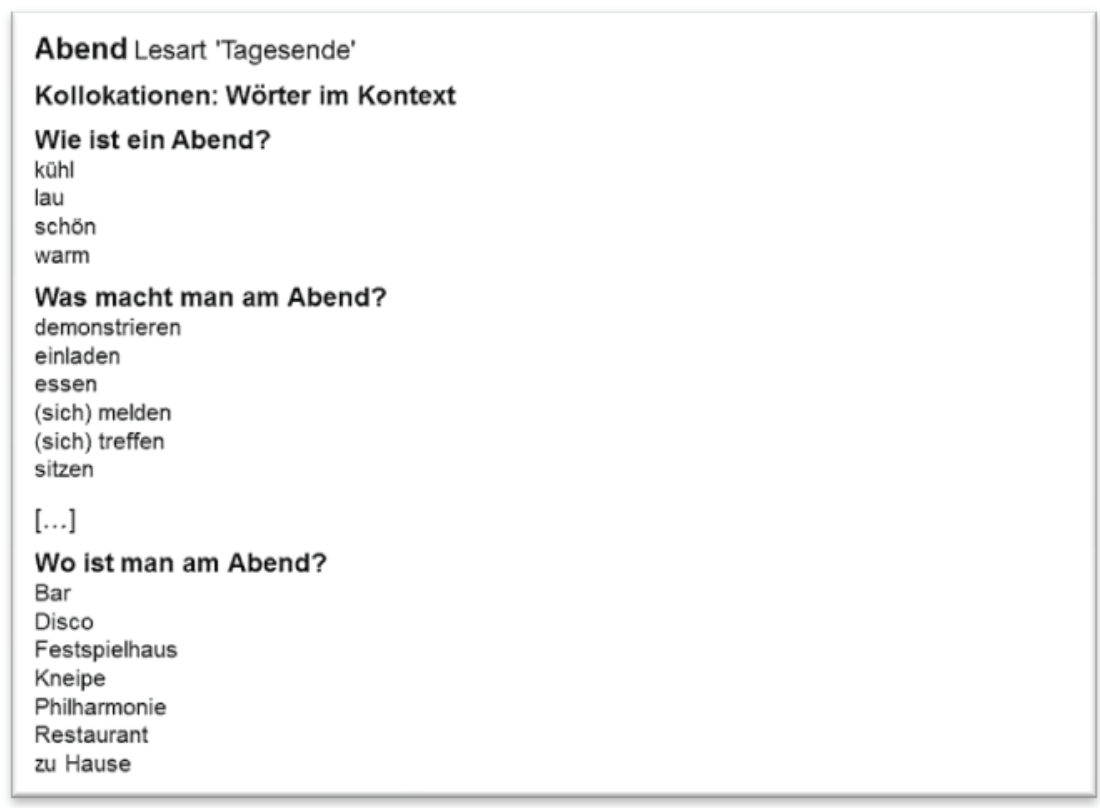

Abb. 4: Auszug aus den verschiedenen Kollokationsgruppen im Angabebereich „Kollokationen“ zum Stichwort Abend, Lesart ,Tagesende“ (elexiko)

Auch wenn zur Zeit der Konzeption und der Erarbeitung der ersten Wortartikel die Funktion für Nutzer und Nutzungssituationen noch nicht ausdifferenziert feststand, war in jedem Fall mitgedacht, dass Nutzer beim Konsultieren dieses Angabebereiches zu einem Stichwort „etwas Interessantes über Wörter und Wortschatz lernen“ (Haß 2005b, S. 227) können. Als genau solch eine Wort-Datenbank wird dieser Angabebereich in diesem Beitrag angenommen.

13 Bei der Darstellung handelt es sich um eine der Onlineansicht nachempfundene Darstellung, die exemplarisch Auszüge aus den Sets aus Fragen mit zugehörigen Antworten zeigt. Die vollständige Ansicht zum Stichwort Abend, Lesart ,Tagesende', ist einzusehen unter www.owid.de/artikel/269290/Tagesende?module=elex_b. 
3.2 Wortschatzübungen anhand von Kollokationsangaben aus elexiko

Die vor allem semantische Gruppierung der Kollokatoren im Wörterbuch elexiko ermöglicht es einem Lehrenden, auf Kollokatoren zu einem Stichwort (in einer bestimmten Lesart) mit einem bestimmten inhaltlichen Schwerpunkt zuzugreifen. In Tabelle 1 sind drei inhaltlich vergleichbare Frage-Antworten-Sets aus den Wortartikeln morgens, mittags und abends im Vergleich zusammengestellt, die zur Konzipierung einer Wortschatzübung, in der es um Essgewohnheiten geht, herangezogen werden können. Da das elexiko-Korpus aus (Zeitungs-)Texten aus dem deutschsprachigen Raum zusammengesetzt ist, kann an der Zusammenstellung eine gewisse Kulturspezifik abgelesen werden. Die Vielfältigkeit bei den Kollokatoren lässt vermuten, dass die Mahlzeit am Mittag die Hauptmahlzeit ist. Den Kollokatoren (aus dem elexiko-Korpus) nach zu urteilen (,Was isst und trinkt man ...?“ bzw. „Was gibt es ... zu essen und zu trinken?“), fällt die Mahlzeit am Mittag sehr viel umfänglicher als morgens und abends aus.

\begin{tabular}{|c|c|c|}
\hline $\begin{array}{c}\text { Was isst und trinkt man } \\
\text { morgens? }\end{array}$ & $\begin{array}{c}\text { Was gibt es mittags zu essen } \\
\text { und zu trinken? }\end{array}$ & $\begin{array}{c}\text { Was isst und trinkt man } \\
\text { abends? }\end{array}$ \\
\hline Brötchen & Bier & Bier \\
Frühstück & Brötchen & Bierchen \\
Kaffee & Eintopf & Essen \\
& Essen & Mahlzeit \\
& Imbiss & Wein \\
& Kaffee & \\
Lunch & \\
& Mahlzeit & \\
& Menü & \\
& Obst & \\
& Pommes & \\
& Salat & \\
& Schnitzel & \\
& Spaghetti & \\
& Suppe & \\
&
\end{tabular}

Tab. 1: Frage-Antworten-Sets aus dem Angabebereich „Kollokationen“ zu den Stichwörtern morgens, mittags, abends (elexiko)

In einer konkreten Unterrichtssituation könnte die Lehrperson zunächst kurz das Wörterbuch elexiko vorstellen und auf den Angabebereich „Kollokationen“ hinweisen. Um die folgende Wortschatzübung in Form eines Lückentextes zu bearbeiten, könnten die Schüler gebeten werden, die Angaben zu den in der Aufgabenstellung genannten Stichwörtern in den entsprechenden Wortartikeln in elexiko nachzuschlagen und auf dieser Basis den Lückentext zu ergänzen (vgl. Abb. 5). 


\section{Was wird morgens, mittags und abends gegessen? Beschreibe typische Essgewohnheiten.}

Morgens isst man

Mittags gibt es zu essen.

Abends trinkt man

Abb. 5: Wortschatzübung zu typischen Essgewohnheiten

Betrachtet man die Kollokatoren im Frage-Antwort-Set „Was gibt es am bzw. in der ...?“, können im Vergleich ebenfalls Gewohnheiten und übliche Ereignisse zu bestimmten Tageszeiten herausgelesen werden (vgl. Tab. 2). Anhand der Kollokatoren kann beispielsweise ein typischer Tagesablauf nachgezeichnet werden, wie er in den Texten des elexikoKorpus thematisiert wird: am Morgen $=$ Berufsverkehr, am Mittag = Pause, am Abend = Party, in der Nacht $=$ Schlaf .

\begin{tabular}{|c|c|c|c|}
\hline $\begin{array}{l}\text { Was gibt es am } \\
\text { Morgen? }\end{array}$ & $\begin{array}{c}\text { Was gibt es am } \\
\text { Mittag? }\end{array}$ & $\begin{array}{c}\text { Was gibt es am } \\
\text { Abend? }\end{array}$ & $\begin{array}{c}\text { Was gibt es in der } \\
\text { Nacht? }\end{array}$ \\
\hline $\begin{array}{l}\text { Berufsverkehr } \\
\text { Frühstück } \\
\text { Journal } \\
\text { Muskelkater } \\
\text { Nebel } \\
\text { Sonne } \\
\text { Tageszeitung } \\
\text { Wecker }\end{array}$ & $\begin{array}{c}\text { Essen } \\
\text { Kaffee } \\
\text { Lunch } \\
\text { Mahlzeit } \\
\text { Menü } \\
\text { Pause } \\
\text { Picknick } \\
\text { Pressekonferenz } \\
\text { Quellwolken } \\
\text { Rast } \\
\text { Regen } \\
\text { Regenschauer } \\
\text { Schweigeminute } \\
\text { Sonne } \\
\text { Suppe } \\
\text { Unfall } \\
\text { Wolken }\end{array}$ & $\begin{array}{c}\text { Bankett } \\
\text { Bier } \\
\text { Dinner } \\
\text { Dunkelheit } \\
\text { Feuerwerk } \\
\text { Gewitter } \\
\text { Kino } \\
\text { Konzert } \\
\text { Lagerfeuer } \\
\text { Party } \\
\text { Prosecco } \\
\text { Schauer } \\
\text { Wein } \\
\text { Wolkenfelder }\end{array}$ & $\begin{array}{c}\text { Feuer } \\
\text { Hitze } \\
\text { Regen } \\
\text { Sachschaden } \\
\text { Schlaf } \\
\text { Schüsse } \\
\text { Temperaturen }\end{array}$ \\
\hline
\end{tabular}

Tab. 2: Frage-Antwort-Sets aus dem Angabebereich „Kollokationen“ zu den Stichwörtern Morgen, Mittag, Abend und Nacht (in den Lesarten, die Tageszeiten beschreiben) (elexiko)

$\mathrm{Zu}$ den Gewohnheiten und Ereignissen zu bestimmten Tageszeiten könnte eine Wortschatzübung wie in Abbildung 6 entstehen, in der die Lernenden die passenden Vorkommnisse und Ereignisse zu den Tageszeiten finden und entsprechend zuordnen sollen. 


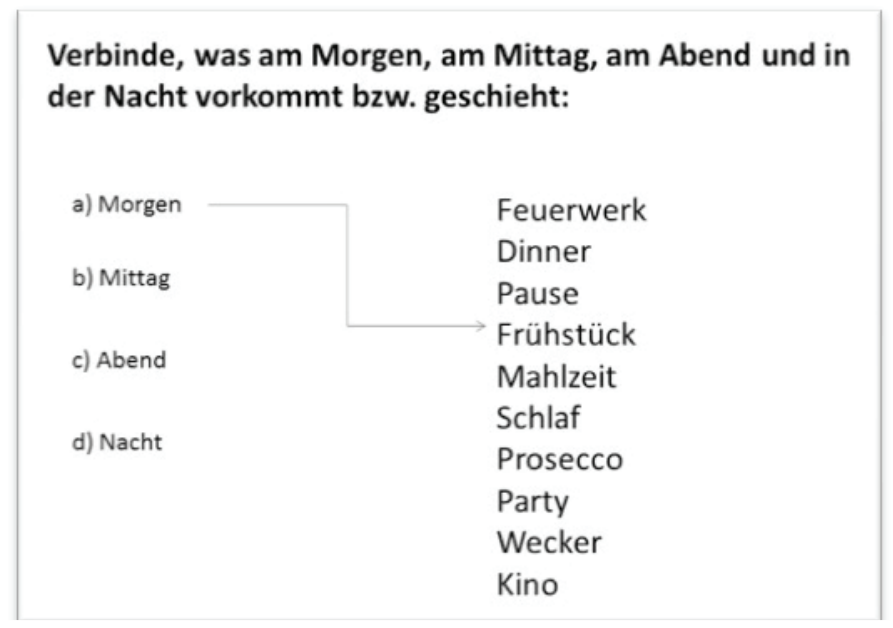

Abb. 6: Wortschatzübung zu Tageszeiten mit Kollokationen zu den Stichwörtern Morgen, Mittag, Abend und Nacht (in den Lesarten, die Tageszeiten beschreiben) (elexiko)

Bei substantivischen Stichwörtern gibt es in den elexiko-Wortartikeln in der Regel ein Frageset „Wie ist ...?", das typische Adjektivkollokatoren des substantivischen Stichworts aufführt. Vergleich man dieses Frageset in den verschiedenen Wortartikeln der Bezeichnungen für die Tageszeiten, so fällt auf, dass die in Tabelle 3 aufgelisteten Adjektive vor allem die meteorologischen Bedingungen zur jeweiligen Tageszeit charakterisieren (z.B. eisig, lau, neblig, sonnig, schwül, verregnet, warm). Das Stichwort Nacht zeigt aber auch Adjektivkollokatoren, welche die Nacht in ihrer Ereignishaftigkeit charakterisieren (z.B. durchwacht, durchzecht, schlaflos, unvergesslich) und somit auch kulturelle Aspekte thematisieren.

\begin{tabular}{|c|c|c|c|c|}
\hline $\begin{array}{c}\text { Wie ist ein } \\
\text { Morgen? }\end{array}$ & $\begin{array}{c}\text { Wie ist ein } \\
\text { Mittag? }\end{array}$ & $\begin{array}{c}\text { Wie ist ein } \\
\text { Nachmittag? }\end{array}$ & $\begin{array}{c}\text { Wie ist ein } \\
\text { Abend? }\end{array}$ & $\begin{array}{c}\text { Wie ist eine } \\
\text { Nacht? }\end{array}$ \\
\hline gut & bewölkt & ereignisreich & kühl & bitterkalt \\
neblig & hell & frei & lau & dunkel \\
sonnig & helllicht & hell & schön & durchwacht \\
& sonnig & ruhig & warm & durchzecht \\
& & schön & & eisig \\
& schwül & & frostig \\
& sonnig & & heiß \\
& & & kalt \\
& & & & lau \\
& & & & ruhig \\
& & & & schlaflos \\
& & & & sternenklar \\
& & & & stürmisch \\
& & & & unruhig \\
& & & & unvergesslich \\
\hline
\end{tabular}

Tab. 3: Frage-Antwort-Sets aus dem Angabebereich „Kollokationen“ zu den Stichwörtern Morgen, Mittag, Nachmittag, Abend und Nacht (in den Lesarten, die Tageszeiten beschreiben) (elexiko) 
Typische Adjektivkollokatoren, wie in Tabelle 3 zu den Bezeichnungen für die Tageszeiten gezeigt, können nicht nur für Wortschatzübungen verwendet, sondern auch für grammatische Übungen genutzt werden. Abbildung 7 zeigt eine Übung, mit der die (starke und schwache) Flexion des Adjektivs in attributiver Stellung trainiert werden kann. Durch die Verwendung bestimmter Adjektivkollokatoren zu den einzelnen Tageszeitenbezeichnungen aus Tabelle 3 werden indirekt auch semantische Aspekte vermittelt.

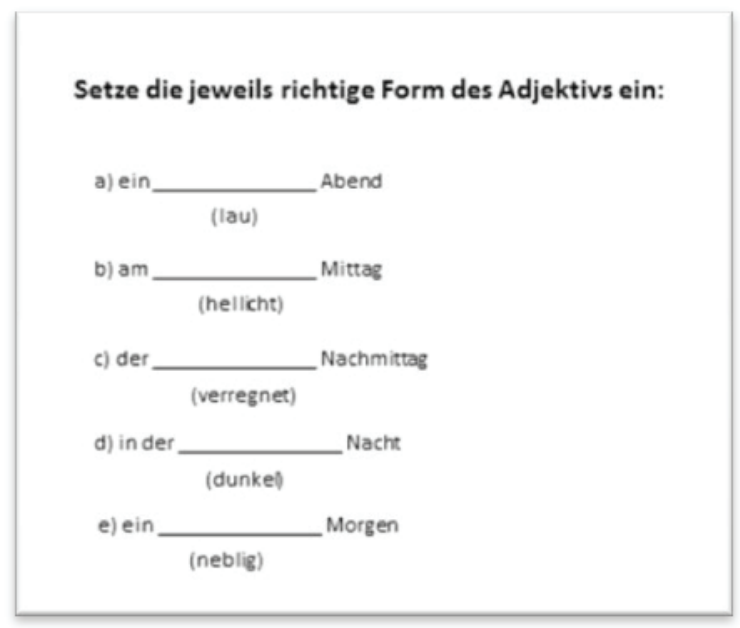

Abb. 7: Übung zur Adjektivflexion unter Verwendung typischer Adjektivkollokatoren aus den Stichwörtern Morgen, Mittag, Nachmittag, Abend und Nacht (in den Lesarten, die Tageszeiten beschreiben) (elexiko)

Eine weitere denkbare Übung, die sich aber nur an Lerner ab Niveau C1 wenden dürfte, könnte sowohl Landeskunde und daneben auch freies Formulieren in den Vordergrund stellen. Die Lehrperson könnte z.B. alle Kollokationen (oder eine geschickte Auswahl) zum Artikel Abend (Lesart ,Tagesende') zeigen (also Adjektiv-, Verb-, Substantivkollokatoren auf der Basis der Angaben aus elexiko) und die Schüler bitten, aus diesen jeweils einen Satz zu bilden, z.B. „Am Abend trifft man sich in der Kneipe.“ oder „Wir sind zu einem Bier oder Wein am Abend eingeladen.“ oder „Wenn es am Abend warm ist, gibt es auch oft Gewitter.“. Man könnte auch im Unterricht Ausschnitte aus den jeweiligen Tageszeiten-Lesarten zu Morgen, Mittag etc. oder den Adverbien wie abends, nachts etc. zeigen und mit den Schülern gemeinsam erarbeiten, was jeweils am Morgen, am Abend, in der Nacht passiert. Eine denkbare Wortschatzübung könnte dann sein, dass die Schüler nach weiteren Veranstaltungen suchen, die z.B. abends stattfinden, oder weitere Getränke/ Gerichte sammeln, die man zu bestimmten Tageszeiten trinkt/isst. In gemeinschaftlicher Interaktion könnte das Thema ,Zeit‘ erweitert werden. Im Gespräch könnten dabei auch kulturelle Unterschiede thematisiert werden (was isst man z.B. in Brasilien oder in Italien am Mittag).

\section{Schlussbemerkungen}

Zusammenfassend kann festgehalten werden, dass die Wortschatzarbeit nach der ,Kognitiven Wende' (vgl. Ulrich 2011, S. 9) wieder stärker in das Bewusstsein gerückt ist und als wichtiger Baustein für den Sprachunterricht eingestuft wird (vgl. auch die Beiträge von Storjohann und Töpel in diesem Band). Je nach Unterrichtskonzept wird die Arbeit am Wortschatz in impliziter oder expliziter Form umgesetzt. Mit Hilfe der Informationen aus dem Angabebereich „Kollokationen“ im Wörterbuch elexiko können (lesartenbezo- 


\section{Christine Möhrs}

gen) Wortschatzübungen konzipiert werden, wie in Kapitel 3.2 kurz skizziert wurde. Besonders die nach semantischen Gesichtspunkten gruppierten Kollokationen (vgl. Haß 2005b; Klosa/Storjohann 2011) im Onlinewörterbuch elexiko stellen einen reichhaltigen Schatz an Vokabular zur Verfügung, der bei der Konzipierung von Wortschatzübungen herangezogen werden kann.

Neben sprach- und wortschatzbezogenem Wissen für Lernende des Deutschen als Zweitbzw. Fremdsprache ist es möglich, über bestimmte Themenfelder sprachbezogenes, landeskundliches Wissen mit einfließen zu lassen und in den Wortschatzübungen zu verarbeiten. Dabei kann ein Deutschlehrender bei der Konzeption von Wortschatzübungen bei Konsultation eines Wörterbuches wie elexiko auf relevantes, authentisches Sprachmaterial zurückgreifen. Die exemplarische Erprobung zeigt, dass darin Potenzial stecken kann. Die Betrachtung in diesem Beitrag war exemplarisch auf Tageszeiten gerichtet, könnte aber auf andere (Zeit-)Bereiche (z.B. Wochentage, Jahreszeiten) oder Themenfelder ${ }^{14}$ (deutsche Städte, Einrichtungsgegenstände etc.) angewendet werden. Um noch stärker landeskundliche Aspekte sprachvergleichend herauszuarbeiten, wäre der Vergleich zu Stichwörtern und ihren Kollokationen aus einem ebenfalls korpusgestützt erarbeiteten Wörterbuch zum Englischen denkbar, z.B. dem „Oxford Collocations Dictionary“. Hier ist als Kollokator zum Stichwort Morgen beispielsweise Tee aufgeführt (im Vergleich zu elexiko, wo Kaffee verzeichnet ist).

Zieht eine Lehrperson prinzipiell korpusgestützt ermittelte Wörterbuchangaben zu Rate, wenn sie Wortschatzübungen zu sprachbezogener Landeskunde konzipiert, so wäre in Hinblick auf die Kollokationen gewährleistet, dass diese ein thematisches Feld so abdecken, dass die inhaltlich zusammengehörenden und als typisch einzustufenden Kontexte vorkommen und der behandelte Wortschatz nicht willkürlich ausgewählt ist.

\section{Literatur}

Belica, Cyril (1995): Statistische Kollokationsanalyse und -clustering. COSMAS-Korpusanalysemodul. Internet: www1.ids-mannheim.de/kl/projekte/methoden/ka.

Bettermann, Rainer (2010): Sprachbezogene Landeskunde. In: Krumm, Hans-Jürgen et al. (Hg.): Deutsch als Fremd- und Zweitsprache. Ein internationales Handbuch. Berlin/New York: de Gruyter Mouton. S. 1454-1465. (= Handbücher zur Sprach- und Kommunikationswissenschaft 35.2).

Eichinger, Ludwig M. (2008): Wer zur Sprache etwas zu sagen hat. Sprachwissenschaft zwischen Natur und Kultur. In: Kämper, Heidrun/Eichinger, Ludwig M. (Hg.): Sprache, Kognition, Kultur: Sprache zwischen mentaler Struktur und kultureller Prägung. Berlin u.a.: de Gruyter. S. 1-13.

Geyken, Alexander (2011a): Statistische Wortprofile zur schnellen Analyse der Syntagmatik in Textkorpora. In: Abel, Andrea/Zanin, Renata (Hg.): Korpora in Lehre und Forschung. Bozen: Bolzano University Press. S. 115-137.

Geyken, Alexander (2011b): Die dynamische Verknüpfung von Kollokationen mit Korpusbelegen und deren Repräsentation im DWDS-Wörterbuch. In: Klosa, Annette/Müller-Spitzer, Carolin (Hg.): Datenmodellierung für Internetwörterbücher. 1. Arbeitsbericht des wissenschaftlichen Netzwerks „Internetlexikografie“. Mannheim: Institut für Deutsche Sprache. S. 9-22.

Grucza, Franciszek (2000): Kultur aus der Sicht der Angewandten Linguistik. In: Schlosser, Horst Dieter (Hg.): Sprache und Kultur. Frankfurt a.M. u.a.: Lang, S. 17-29. (= forum Angewandte Linguistik 38).

Häcki Buhofer, Annelies et al. (2014): Feste Wortverbindungen des Deutschen. Kollokationenwörterbuch für den Alltag. Tübngen: Francke.

\footnotetext{
14 Zu der Beschreibung von Wortfeldern in Wörterbüchern vgl. auch den Beitrag von Schnörch in diesem Band.
} 
Hansen, Klaus P. (2000): Kultur und Kulturwissenschaft. Eine Einführung. 2., vollständig überarbeitete und erweiterte Auflage. Tübingen/Basel: Francke.

Haß, Ulrike (Hg.) (2005): Grundfragen der elektronischen Lexikographie. elexiko - das Online-Informationssystem zum deutschen Wortschatz. Berlin/New York: de Gruyter. (= Schriften des Instituts für Deutsche Sprache 12).

Haß, Ulrike (2005a): elexiko - das Projekt. In: Haß, Ulrike (Hg.), S. 1-17.

Haß, Ulrike (2005b): Semantische Umgebung und lexikalische Mitspieler. In: Haß, Ulrike (Hg.) (2005a), S. 227-233.

Hochstadt, Christiane/Krafft, Andreas/Olsen, Ralph (2013): Deutschdidaktik. Konzeptionen für die Praxis. Tübingen/Basel: Francke. (= UTB 4023).

Klosa, Annette (Hg.) (2011): elexiko. Erfahrungsberichte aus der lexikographischen Praxis eines Internetwörterbuchs. Tübingen: Narr. (= Studien zur Deutschen Sprache 55).

Klosa, Annette/Storjohann, Petra (2011): Neue Überlegungen und Erfahrungen zu den lexikalischen Mitspielern. In: Klosa, Annette (Hg.), S. 49-80.

Koreik, Uwe (2011): Zur Entwicklung der Landeskunde bzw. der Kulturstudien im Fach DaF/DaZ. Was haben wir erreicht, mit welchen Widersprüchen kämpfen wir und wie geht es weiter? In: Info DaF 6, S. 581-604.

Maijala, Minna (2009): Landeskunde im Schatten der Sprache - Betrachtungen aus Forschung und Praxis. In: Di Meola, Claudio et al. (Hg.): Perspektiven Drei. Akten der 3. Tagung Deutsche Sprachwissenschaft in Italien (Rom, 14.-16.2.2008). Frankfurt a.M. u.a.: Lang. S. 373-383.

Meier, Stefanie/Rösch, Eva/Runte Caroline unter Mitarbeit von Anna Fischer (2014): Feste Wortverbindungen des Deutschen. Beiheft für Selbststudium und Unterricht. Tübingen: Francke.

Meier, Stefanie/Widmer, Alessandra (2014): Kollokationsübungen für den Unterricht als Wörterbuchergänzung. In XVI EURALEX International Congress: The User in Focus. 15-19 July 2014. Bolzano/Bozen. Book of Abstract, S. 24. Internet: http://euralex2014.eurac.edu/en/programme/Documents/Book_of_ Abstracts.pdf.

Müller, Christina M./Siever, Torsten (2011): Neue Medien und Wortschatzarbeit. In: Esterl, Ursula/Struger, Jürgen (Hg.): Wort.Schatz - Wörter.schätzen. Innsbruck/Wien/Bozen: StudienVerlag, S. 42-53. (= ide. informationen zur deutschdidaktik. Zeitschrift für den Deutschunterricht in Wissenschaft und Schule 35).

Petelin, Stefanie (2011): Wort.Schatz. Bibliographische Notizen für den Deutschunterricht. In: Esterl, Ursula/Struger, Jürgen (Hg.): Wort.Schatz - Wörter.schätzen. Innsbruck/Wien/Bozen: StudienVerlag, S. 112118. (= ide. informationen zur deutschdidaktik. Zeitschrift für den Deutschunterricht in Wissenschaft und Schule 35).

Reder, Anna (2009): Aktuelle Herausforderungen der Wortschatzdidaktik. In: Feld-Knapp, Ilona (Hg.): Deutsch als Fremdsprache. Sprachdidaktische Überlegungen zu Wortschatz und Textkompetenz. Budapest: Ungarischer Deutschlehrerverband. S. 101-125.

Rösler, Dietmar (2012): Deutsch als Fremdsprache. Eine Einführung. Weimar: Metzler.

Simon-Pelanda, Hans (2001): Landeskundliches Lernen und Lehren. In: Helbig, Gerhard et al. (Hg.): Deutsch als Fremdsprache. Ein internationales Handbuch. 2. Halbband. Berlin/New York: de Gruyter. S. 931-942. (= Handbücher zur Sprach- und Kommunikationswissenschaft 19.2).

Storjohann, Petra (2005): Das elexiko-Korpus: Aufbau und Zusammensetzung. In: Haß, Ulrike (Hg.), S. 55-70.

Ulrich, Winfried (2007): Wörter, Wörter, Wörter: Wortschatzarbeit im muttersprachlichen Deutschunterricht: Anleitungen und praktische Übungen mit 204 Arbeitsblättern in Form von Kopiervorlagen. Hohengehren: Schneider.

Ulrich, Winfried (2011): Wortschatzarbeit im Deutschunterricht. In: Esterl, Ursula/Struger, Jürgen (Hg.): Wort.Schatz - Wörter.schätzen. Innsbruck/Wien/Bozen: StudienVerlag, S. 9-21. (= ide. informationen zur deutschdidaktik. Zeitschrift für den Deutschunterricht in Wissenschaft und Schule 35). 


\section{Christine Möhrs}

Veeck, Reiner/Linsmayer, Ludwig (2001): Geschichte und Konzepte der Landeskunde. In: Helbig, Gerhard et al. (Hg.): Deutsch als Fremdsprache. Ein internationales Handbuch. 2. Halbband. Berlin/New York: de Gruyter. S. 1160-1168. (= Handbücher zur Sprach- und Kommunikationswissenschaft 19.2).

\section{Nachschlagewerke und elektronische Ressourcen (Stand 31.7.2014)}

COSMAS II: www.ids-mannheim.de/cosmas2/

DeReKo - Deutsches Referenzkorpus des IDS: www1.ids-mannheim.de/kl/projekte/korpora/

Duden online: www.duden.de/

DWDS - Das Digitale Wörterbuch der deutschen Sprache des 20. Jahrhunderts: www.dwds.de/

elexiko (2003ff.). In: Institut für Deutsche Sprache (Hg.): OWID - Online Wortschatz-Informationssystem Deutsch. Mannheim: www.owid.de/wb/elexiko/start.html.

Europäischer Referenzrahmen: www.europaeischer-referenzrahmen.de/

Kollokationenwörterbuch. Typische und gebräuchliche Wortverbindungen des Deutschen: http://colloc. germa.unibas.ch/web/suche/

Konzepte und Materialien. Deutsche Sprache. Goethe- Institut - Sprache. Kultur. Deutschland. Internet: www.goethe.de/de/spr/unt/kum.html

Oxford collocations dictionary: for students of English. 1. Auflage. Oxford u.a.: Oxford University Press, 2002.

Dr. des. Christine Möhrs

Institut für Deutsche Sprache

R 5, 6-13

D-68161 Mannheim

E-Mail: moehrs@ids-mannheim.de 